Open Access

\title{
Are personality disturbances in anorexia nervosa related to emotion processing or eating disorder symptomatology?
}

\author{
Andrea Phillipou ${ }^{1,2,3,8^{*}}$, Caroline Gurvich ${ }^{4}$, David Jonathan Castle ${ }^{5,2,6}$ and Susan Lee Rossell ${ }^{7,4,5}$
}

\begin{abstract}
Background: Anorexia Nervosa (AN) is a psychiatric illness associated with a number of personality disturbances. However, whether these personality characteristics are related to eating disorder symptomatology or emotion regulation is unclear. The aim of this study was to investigate these relationships.
\end{abstract}

Results: Twenty-four individuals with AN and 25 age- and premorbid intelligence-matched controls completed the Personality Diagnostic Questionnaire, and scores were correlated with measures of emotionality and negative mood states, and eating disorder symptomatology. AN was associated with increased scores on schizoid, borderline, avoidant, dependent, obsessive compulsive, negativistic and depressive personality dimensions, relative to controls. In AN, eating disorder symptomatology did not significantly correlate with scores on any personality dimension. However, a number of personality characteristics were found to correlate with negative mood states.

Conclusions: The findings suggest that personality disturbances in AN are not related to disorder-specific symptoms, but are related to negative mood states.

\section{Background}

Anorexia Nervosa (AN) is a psychiatric illness characterised by an intense fear of weight gain, low body weight, and a disturbance in the experience of one's own body shape or weight. The condition is also frequently associated with a number of personality traits, including perfectionism [1-5], rigid thinking patterns [6] and obsessive compulsive behaviours and thoughts [7].

Relatedly, AN has been associated with high levels of comorbid obsessive compulsive personality disorder, as well as avoidant, borderline and dependent personality disorders [8-11]. Personality disorders are related to poor emotion processing and negative mood states [12, 13], which are similarly reported in AN $[14,15]$. Thus, it is unclear whether personality disturbances in AN interact with eating disorder symptomatology, or whether they relate to difficulties in emotion regulation. Thus, the aim

\footnotetext{
* Correspondence: ap@unimelb.edu.au

'Department of Optometry \& Vision Sciences, The University of Melbourne, Melbourne, Australia

2Department of Psychiatry, The University of Melbourne, Melbourne, Australia Full list of author information is available at the end of the article
}

of this study was to investigate these relationships in AN. Furthermore, given the lack of current treatment options for AN [16], this research is warranted to determine which personality variables are associated with mood regulation or eating disorder symptomatology to help guide the development of more tailored and effective interventions for AN.

It was hypothesised that the AN group would demonstrate higher scores on a number of personality disturbances relative to healthy controls, namely, obsessive compulsive, avoidant, borderline and dependent personality disorders. Given the lack of research investigating relationships of other psychological processes with personality disturbances in AN, the primary aim of this study was exploratory, to examine whether personality disturbances in AN relate to mood states or emotion regulation within the self (i.e. alexithymia) and eating disorder symptomatology.

\section{Method}

This study was approved by the human research ethics departments at The University of Melbourne, Swinburne University of Technology, The Melbourne Clinic, The 
Austin Hospital and St Vincent's Hospital; all in Melbourne, Australia. Informed written consent was obtained from all participants. The authors assert that all procedures contributing to this work comply with the ethical standards of the relevant national and institutional committees on human experimentation and with the Helsinki Declaration of 1975, as revised in 2008.

\section{Participants}

Participants were 24 right-handed females with AN and 25 healthy controls $(\mathrm{HC})$ matched for age and premorbid intelligence quotient (IQ) to ensure comparability between groups. HCs were recruited through public advertisements, whereas AN participants were recruited through public advertisements; the Body Image and Eating Disorders Treatment and Recovery Service at the Austin and St Vincent's Hospitals; and The Melbourne Clinic; all in Melbourne, Australia. The Austin and St Vincent's Hospitals are public hospitals accepting referrals from Northern, Eastern and Hume regions of Melbourne. The Melbourne Clinic is a private mental health service, and unlike the public health services, only accepts patients on a voluntary basis.

All participants were English speaking and had no history of significant brain injury or neurological condition. Controls were required to have no history of an eating disorder or other mental illness. The Mini International Neuropsychiatric Interview, 5.0.0 (MINI) [17] was used to screen all participants for major Axis I psychiatric disorders according to the Diagnostic and Statistical Manual of Mental Disorders (DSM-IV). It was also used to confirm diagnoses of AN, with the exception of the amenorrhea criterion which is no longer included in the current DSM-5. $\mathrm{AN}$ was required to be the primary diagnosis of the AN group. AN participants with comorbid psychiatric conditions, other than psychotic conditions, were not excluded as this would not have represented a typical AN sample. The following comorbid conditions were identified in the AN group: current depressive episode $(\mathrm{n}=17)$, dysthymia $(n=14)$, current manic/hypomanic episode $(n=2)$, current panic disorder $(n=3)$, social anxiety disorder $(n=8)$, obsessive compulsive disorder $(n=12)$, posttraumatic stress disorder $(\mathrm{n}=4)$, alcohol dependence $(\mathrm{n}=2)$, substance dependence $(n=1)$, bulimia nervosa $(n=3)$, generalised anxiety disorder $(\mathrm{n}=17)$.

Premorbid intelligence was estimated using the Wechsler Test of Adult Reading (WTAR) [18] and was used to ensure all participants had an IQ over 80, to ensure they could adequately comprehend the study instructions and correctly complete the questionnaires. Eating disorder symptomatology was investigated with the Eating Disorders Examination Questionnaire (EDE-Q) [19] (Table 1), and negative emotional states with the Depression Anxiety Stress Scale (DASS) [20]. The alexithymia construct was measured with the Toronto Alexithymia Scale (TAS-20) [21], and personality psychopathology with the Personality Diagnostic Questionnaire (PDQ-4) [22]. The PDQ-4 allows for the examination of continuous symptom scores for each personality disorder and categorical scoring (i.e. presence or absence) of clinically significant personality disorders.

\section{Statistical analyses}

Following normality checking and the removal of outliers (identified as cases lying outside more than 1.5x the interquartile range, through Tukey's boxplots), group differences in EDE-Q, DASS and TAS-20 scores were examined with analyses of variance (ANOVAs). Scores on the PDQ-4 were not normally distributed, thus Mann-Whitney tests were utilised. The number of clinically significant personality disorders as identified by the PDQ- 4 was also compared between groups with $\chi^{2}$ tests. Pearson's correlations were also conducted, following outlier removal (as above), between PDQ scores and the other measures for AN and HC groups, these included DASS, TAS and EDE-Q. Due to the number of correlational analyses, alpha was set at 0.001 to account for multiple comparisons, and trends were identified as alpha $<0.01$.

\section{Results}

AN participants scored significantly higher on total PDQ score, as well as items related to schizoid, borderline, avoidant, dependent, obsessive compulsive, negativistic and depressive personality disorders (Table 2). Whether the items reached clinical significance was also explored and the results are detailed in Table 3. AN participants were more likely than control participants to have a clinically significant personality disorder $\left(x^{2}(1)=22.40, p<0.001\right)$.

\section{Correlations with DASS subscales}

In the AN group, significant positive correlations were found between schizoid personality scores and depression $(r=0.62, p<0.001)$, avoidant personality scores and depression $(\mathrm{r}=0.64, \mathrm{p}<0.001)$, and borderline personality and stress $(r=0.62, p<0.001)$. Trends were identified between paranoid personality scores and stress $(r=0.52, p=0.009)$, dependent personality scores and both depression $(r=0.56$, $\mathrm{p}=0.004)$ and stress $(\mathrm{r}=0.56, \mathrm{p}=0.005)$, and between depressive personality scores and stress $(r=0.59, p=0.002)$. In the $\mathrm{HC}$ group, no significant correlations were found between personality scores and DASS subscales. A trend was however identified between paranoid personality scores and anxiety scores $(\mathrm{r}=0.53, \mathrm{p}=0.008)$.

\section{Correlations with TAS-20 subscales}

In the AN group, no significant correlations were observed, however, a positive trend was found between schizotypal personality scores and the difficulty describing 
Table 1 Participant information

\begin{tabular}{|c|c|c|c|c|c|}
\hline & \multicolumn{2}{|l|}{ AN } & \multicolumn{3}{|l|}{$\mathrm{HC}$} \\
\hline & M & SD & M & SD & $p$ \\
\hline Age & 23.07 & 6.88 & 22.67 & 3.19 & 0.798 \\
\hline Premorbid IQ & 104.67 & 8.19 & 105.60 & 7.00 & 0.670 \\
\hline BMl & 16.52 & 1.14 & 22.40 & 3.59 & 0.001 \\
\hline Illness duration & 6.67 & 7.66 & - & - & - \\
\hline Age of illness onset & 16.04 & 3.50 & - & - & - \\
\hline EDE-Q restraint & 3.93 & 1.42 & 0.43 & 0.40 & 0.001 \\
\hline EDE-Q eating concern & 3.78 & 1.24 & 0.20 & 0.20 & 0.001 \\
\hline EDE-Q shape concern & 5.01 & 0.90 & 0.99 & 0.59 & 0.001 \\
\hline EDE-Q weight concern & 4.50 & 1.41 & 0.42 & 0.47 & 0.001 \\
\hline EDE-Q global score & 4.30 & 1.12 & 0.60 & 0.43 & 0.001 \\
\hline DASS depression & 25.08 & 12.41 & 1.08 & 1.29 & 0.001 \\
\hline DASS anxiety & 16.00 & 9.48 & 1.88 & 2.13 & 0.001 \\
\hline DASS stress & 24.92 & 10.23 & 3.78 & 2.78 & 0.001 \\
\hline TAS-20 difficulty identifying feelings & 22.71 & 5.76 & 10.65 & 3.70 & 0.001 \\
\hline TAS-20 difficulty describing feelings & 18.43 & 2.74 & 10.64 & 4.05 & 0.001 \\
\hline TAS-20 externally oriented thinking & 20.42 & 3.32 & 16.78 & 4.65 & 0.003 \\
\hline TAS-20 score & 61.17 & 8.86 & 38.88 & 11.79 & 0.001 \\
\hline
\end{tabular}

AN Anorexia Nervosa, HC healthy controls, Premorbid IQ standardised Wechsler Test of Adult Reading Score, BMI body mass index, EDE-Q Eating Disorders Examination Questionnaire, DASS Depression, Anxiety, Stress Scale, TAS-20 Toronto Alexithymia Scale, Age age of illness onset and duration illness are reported in years

feelings subscale of the TAS-20 $(\mathrm{r}=0.62, \mathrm{p}=0.002)$. No significant correlations or trends were observed between TAS-20 and personality scores for HCs.

\section{Correlations with EDE-Q subscales}

In the AN group, no significant correlations were observed between EDE-Q subscales and the different personality constructs. However, schizoid personality scores were associated with a number of trends, including, the restraint and eating concern subscales of the EDE-Q $(\mathrm{r}=0.59, \mathrm{p}=0.002 ; \mathrm{r}=0.53, \mathrm{p}=0.008)$, and global EDE-Q scores $(r=0.57, p=0.003)$. In the $\mathrm{HC}$ group, one significant correlation was found between EDE-Q and personality scores: shape concern was positively correlated with

Table 2 Personality diagnostic questionnaire scores

\begin{tabular}{|c|c|c|c|c|c|c|c|}
\hline & \multicolumn{2}{|l|}{ AN } & \multicolumn{2}{|l|}{$\mathrm{HC}$} & \multirow[b]{2}{*}{ U } & \multirow[b]{2}{*}{ z } & \multirow[b]{2}{*}{$p$} \\
\hline & Median & Range & Median & Range & & & \\
\hline Paranoid & 3.0 & 6.0 & 1.0 & 5.0 & 216.5 & -1.7 & 0.087 \\
\hline Schizoid & 2.5 & 6.0 & 0.0 & 4.0 & 102.2 & -4.0 & 0.001 \\
\hline Schizotypal & 2.0 & 4.0 & 1.0 & 5.0 & 234.0 & -1.4 & 0.176 \\
\hline Histrionic & 2.0 & 5.0 & 2.0 & 4.0 & 258.5 & -0.9 & 0.389 \\
\hline Narcissistic & 1.0 & 5.0 & 2.0 & 5.0 & 223.0 & -1.6 & 0.112 \\
\hline Borderline & 4.5 & 7.0 & 1.0 & 3.0 & 41.5 & -5.2 & 0.001 \\
\hline Antisocial & 1.0 & 5.0 & 1.0 & 2.0 & 222.0 & -1.6 & 0.102 \\
\hline Avoidant & 6.0 & 5.0 & 2.0 & 4.0 & 38.5 & -5.3 & 0.001 \\
\hline Dependent & 4.0 & 7.0 & 1.0 & 3.0 & 48.0 & -5.1 & 0.001 \\
\hline Obsessive compulsive & 5.0 & 7.0 & 3.0 & 5.0 & 158.5 & -2.9 & 0.004 \\
\hline Negativistic & 2.0 & 6.0 & 1.0 & 4.0 & 148.5 & -3.1 & 0.002 \\
\hline Depressive & 5.5 & 4.0 & 2.0 & 7.0 & 50.0 & -5.1 & 0.001 \\
\hline Total PDQ score & 38.0 & 47.0 & 19.0 & 26.0 & 51.5 & -5.0 & 0.001 \\
\hline
\end{tabular}

$P D Q$ personality diagnostic questionnaire, $A N$ anorexia nervosa, $H C$ healthy control 
Table 3 Number of clinically significant personality disorders

\begin{tabular}{lll}
\hline & AN & $\mathrm{HC}$ \\
\hline Paranoid & 3 & 0 \\
Schizoid & 4 & 0 \\
Schizotypal & 0 & 0 \\
Histrionic & 0 & 0 \\
Narcissistic & 0 & 1 \\
Borderline & 6 & 0 \\
Antisocial & 0 & 0 \\
Avoidant & 13 & 1 \\
Dependent & 5 & 0 \\
Obsessive compulsive & 13 & 3 \\
Negativistic & 2 & 0 \\
Depressive & 13 & 0 \\
Any personality disorder & 21 & 5 \\
\hline AN anoxa nesa HCheathy contol
\end{tabular}

$A N$ anorexia nervosa, $H C$ healthy control

negativistic personality scores $(\mathrm{r}=0.64, \mathrm{p}<0.001)$. A trend for higher eating concerns was also identified with greater avoidant personality scores in HCs $(0.58, \mathrm{p}=0.003)$.

\section{Discussion}

The aim of this study was to examine personality disturbances in $\mathrm{AN}$, and their relationship to eating disorder symptomatology, negative mood states and alexithymia. As hypothesised, the AN group were found to score significantly higher on a number of personality disturbances. However, the personality constructs in which groups significantly differed were not entirely as expected.

As predicted, the AN group displayed greater scores on obsessive compulsive, avoidant, dependent and borderline personality disorders. In addition, AN participants also scored significantly higher on schizoid, negativistic and depressive personality disturbances, which is consistent with a number of characteristics reported in individuals with AN such as reduced interest in friends and activities, and depressive symptoms $[15,23]$.

There were no significant correlations identified between any of the different personality dimensions and eating disorder symptomatology, or alexithymia in AN. This was not predicted, and differs to a number of studies which have suggested a relationship between personality constructs and eating disorder symptoms in AN (see [10] for a review). However, unlike the current study, other research has tended to focus on personality traits such as perfectionism and obsessionality rather than disturbances in defined personality dimensions. A number of significant relationships were, however, observed for negative mood states. Positive correlations in the AN group were found between both schizoid and avoidant personality dimensions and depression, and between borderline personality disorder scores and stress. These findings suggest that a number of personality disturbances in AN may not be related to disorder-specific symptoms, but to negative mood states / emotionality present in the disorder. One might therefore predict with the stabilisation of mood symptoms, these personality dimensions may normalise in AN.

Of particular interest is the absence of significant correlations between obsessive compulsive personality disorder and eating disorder symptomatology. $\mathrm{AN}$ is frequently associated with obsessive compulsive behaviours and thoughts [7, 24], and the AN group in the current study was similarly found to demonstrate increased symptoms related to obsessive compulsive personality disorder. Though these thoughts and behaviours may represent a core feature of AN, the lack of association with eating disorder symptoms suggests that these features are independent of current AN symptoms. Therefore, increased obsessive compulsive personality symptoms, and the other personality disturbances that were not influenced by negative mood state, may represent stable traits which predispose individuals to developing AN.

Although the current study has resulted in a number of important implications for our understanding of personality disorders in $\mathrm{AN}$, the findings may be limited due to a number of factors. Firstly, the patients recruited for this study were recruited from a specific catchment area or were private hospital patients, thus potentially limiting the generalisability of the sample. However, AN participants were also recruited from public advertisements in an attempt to limit this potential confound. Secondly, the conservative sample size and the number of statistical comparisons undertaken have the potential to have influenced the findings. However, to accommodate this potential confound, the authors applied stringent criteria to indicate significant results, thus limiting the number of false positive errors. An additional limitation of the study is that only acutely ill AN participants were tested, thus a number of findings may reflect state rather than trait effects. As personality disturbances are often reported as a result of starvation [25], future research would benefit from also investigating long term-recovered AN patients whose body weight has restored and negative mood symptoms may have subsided, to examine the stability of personality disturbances in AN. Therefore, a larger sample size, and the inclusion of weight-restored or clinically recovered AN patients would be of benefit in future research. Furthermore, longitudinally assessing the stability of these findings across the different phases of the illness would be beneficial.

\section{Conclusions}

Overall, the findings of the study suggest that individuals with AN exhibit a number of personality disturbances relative to healthy individuals. A number of these personality dimensions were related to negative mood state in 
AN, though none were related to eating disorder symptomatology or emotion processing within the self (alexithymia). Although the findings are limited by the conservative sample size, the results suggest that personality disturbances in AN may not be influenced by disorderspecific symptoms, but may be predisposing factors to developing the illness.

\section{Competing interests}

Prof. Castle reports grants and personal fees from Eli Lilly, grants and personal fees from janssen-Cilag, grants and personal fees from Roche, grants and personal fees from Allergen, grants and personal fees from Bristol-Myer Squibb, grants and personal fees from Pfizer, grants and personal fees from Lundbeck, grants and personal fees from AstraZeneca, grants and personal fees from Hospira, during the conduct of the study; personal fees from Eli Lilly, personal fees from Bristol-Myer Squibb, personal fees from Lundbeck, personal fees from Janssen Cilag, personal fees from Pfizer, personal fees from Organon, personal fees from Sanofi-Aventis, personal fees from Wyeth, personal fees from Hospira, personal fees from Servier, outside the submitted work. Dr Phillipou, Prof Rossell and Dr Gurvich report no conflicts of interest.

\section{Authors' contributions}

All authors were involved in the conception and design of the research, and the interpretation of data. AP collected and analysed the data under the supervision and with the assistance of the other authors. All authors drafted and approved the final manuscript.

\section{Acknowledgements}

This research was supported by the Jack Brockhoff Foundation (S.R., D.C., A.P., grant number: 3410); the Dick and Pip Smith Foundation (A.P., S.R., D.C.); an Australian Postgraduate Award (A.P.) and the David Hay Memorial Fund Award (A.P.). The authors would like to thank Larry Abel for his contribution to the research and assistance in preparing the manuscript, and Charlotte Keating for administering the clinical interview. The authors would also like to thank Chia Huang, Richard Newton, Lynley Gervasoni, Michelle Snell, Helen Shepherd, Philippa Harrison and Felicity Lawrence for their assistance in recruiting participants. Finally, the authors would like to thank everyone who took the time to participate in the study.

\footnotetext{
Author details

'Department of Optometry \& Vision Sciences, The University of Melbourne, Melbourne, Australia. ${ }^{2}$ Department of Psychiatry, The University of Melbourne, Melbourne, Australia. ${ }^{3}$ Department of Mental Health, The Austin Hospital, Melbourne, Australia. ${ }^{4}$ Monash Alfred Psychiatry Research Centre, Melbourne, Australia. ${ }^{5}$ Department of Psychiatry, St Vincent's Hospital, Melbourne, Australia. ${ }^{6}$ Faculty of Health Sciences, Australian Catholic University, Melbourne, Australia. "Brain and Psychological Sciences Research Centre, Swinburne University of Technology, Melbourne, Australia. ${ }^{8}$ Department of Mental Health, St Vincent's Hospital, 46 Nicholson St, Fitzroy, VIC 3065, Australia.
}

Received: 27 July 2015 Accepted: 21 September 2015

Published online: 01 October 2015

\section{References}

1. Bastiani AM, Rao R, Weltzin T, Kaye WH. Perfectionism in anorexia nervosa. Int J Eat Disord. 1995;17(2):147-52.

2. Srinivasagam NM, Kaye WH, Plotnicov KH, Greeno C. Persistent perfectionism, symmetry, and exactness after long-term recovery from anorexia nervosa. Am J Psychiatry. 1995;152(11):1630-4.

3. Halmi KA, Sunday SR, Strober M, Kaplan A, Woodside DB, Fichter M, et al. Perfectionism in anorexia nervosa: variation by clinical subtype, obsessionality, and pathological eating behavior. Am J Psychiatry. 2000;157(11):1799-805.

4. Fairburn CG, Cooper Z, Doll HA, Welch SL. Risk factors for anorexia nervosa: three integrated case-control comparisons. Arch Gen Psychiatry. 1999;56(5):468.

5. Wade TD, Tiggemann M, Bulik CM, Fairburn CG, Wray NR, Martin NG. Shared temperament risk factors for anorexia nervosa: a twin study. Psychosom Med. 2008;70(2):239-44.
6. Steinglass JE, Walsh BT, Stern Y. Set shifting deficit in anorexia nervosa. J Int Neuropsychol Soc. 2006;12(03):431-5.

7. Kaye WH, Weltzin TE, Hsu LK, Bulik C, McConaha C, Sobkiewicz T. Patients with anorexia nervosa have elevated scores on the Yale-Brown obsessivecompulsive scale. Int J Eat Disord. 1992;12(1):57-62.

8. Sansone RA, Levitt JL, Sansone LA. The prevalence of personality disorders among those with eating disorders. Eat Behav. 2004;13(1):7-21.

9. Gillberg IC, Råstam M, Gillberg C. Anorexia nervosa 6 years after onset: Part I. Personality disorders. Compr Psychiatry. 1995;36(1):61-9.

10. Cassin SE, von Ranson KM. Personality and eating disorders: a decade in review. Clin Psychol Rev. 2005;25(7):895-916.

11. Karwautz A, Troop NA, Rabe-Hesketh S, Collier DA, Treasure JL. Personality disorders and personality dimensions in anorexia nervosa. J Pers Disord. 2003;17(1):73-85.

12. Glenn CR, Klonsky ED. Emotion dysregulation as a core feature of borderline personality disorder. J Pers Disord. 2009;23(1):20-8.

13. Brown SL, Svrakic DM, Przybeck TR, Cloninger RC. The relationship of personality to mood and anxiety states: a dimensional approach. J Psychiatr Res. 1992;26(3):197-211.

14. Harrison A, Sullivan S, Tchanturia K, Treasure J. Emotion recognition and regulation in anorexia nervosa. Clin Psychol Psychother. 2009;16(4):348-56.

15. Pollice C, Kaye WH, Greeno CG, Weltzin TE. Relationship of depression, anxiety, and obsessionality to state of illness in anorexia nervosa. Int J Eat Disord. 1997;21 (4):367-76.

16. Hay P, Chinn D, Forbes D, Madden S, Newton R, Sugenor L, et al. Royal Australian and New Zealand College of Psychiatrists clinical practice guidelines for the treatment of eating disorders. Aust N Z J Psychiatry. 2014;48(11):977-1008.

17. Sheehan DV, Lecrubier $Y$, Sheehan $K H$, Amorim P, Janavs J, Weiller E, et al. The Mini-International Neuropsychiatric Interview (MINI): the development and validation of a structured diagnostic psychiatric interview for DSM-IV and ICD-10. J Clin Psychiatry. 1998:59:22-33.

18. Wechsler D. Wechsler Test of Adult Reading: WTAR. San Antonio, TX, USA: Psychological Corporation; 2001.

19. Fairburn CG. Cognitive Behavior Therapy and Eating Disorders. New York: Guilford Press; 2008.

20. Lovibond SH, Lovibond PF. Manual for the Depression Anxiety Stress Scales. Sydney, NSW, Australia: Psychology Foundation of Australia; 1996.

21. Bagby RM, Parker JDA, Taylor GJ. The twenty-item Toronto Alexithymia Scale-I. Item selection and cross-validation of the factor structure. J Psychosom Res. 1994;38(1):23-32.

22. Hyler SE. Personality Diagnostic Questionnaire-4. New York: New York State Psychiatric Institute; 1994.

23. Courty A, Godart N, Lalanne C, Berthoz S. Alexithymia, a compounding factor for eating and social avoidance symptoms in anorexia nervosa. Compr Psychiatry. 2015;56:217-28.

24. Halmi KA, Tozzi F, Thornton LM, Crow S, Fichter MM, Kaplan AS, et al. The relation among perfectionism, obsessive-compulsive personality disorder and obsessive-compulsive disorder in individuals with eating disorders. Int J Eat Disord. 2005;38(4):371-4.

25. Keys A, Brožek J, Henschel A, Mickelsen O, Taylor HL. The biology of human starvation (2 vols). 1950.

\section{Submit your next manuscript to BioMed Central and take full advantage of:}

- Convenient online submission

- Thorough peer review

- No space constraints or color figure charges

- Immediate publication on acceptance

- Inclusion in PubMed, CAS, Scopus and Google Scholar

- Research which is freely available for redistribution 